\title{
Neutrino Oscillations Induced by Two-loop Radiative Mechanism
}

\author{
Teruyuki Kitabayashi $^{a}{ }^{*}$ and Masaki Yasuè ${ }^{b} \dagger$ \\ ${ }^{a}$ Accelerator Engineering Center \\ Mitsubishi Electric System \& Service Engineering Co.Ltd. \\ 2-8-8 Umezono, Tsukuba, Ibaraki 305-0045, Japan \\ ${ }^{b}$ Department of Natural Science \\ School of Marine Science and Technology, Tokai University \\ 3-20-1 Orido, Shimizu, Shizuoka 424-8610, Japan \\ and \\ Department of Physics, Tokai University \\ 1117 KitaKaname, Hiratsuka, Kanagawa 259-1292, Japan
}

(TOKAI-HEP/TH-0001, June, 2000)

\begin{abstract}
Two-loop radiative mechanism, when combined with an $U(1)_{L^{\prime}}$ symmetry generated by $L_{e}-$ $L_{\mu}-L_{\tau}\left(=L^{\prime}\right)$, is shown to provide an estimate of $\Delta m_{\odot}^{2} / \Delta m_{a t m}^{2} \sim \epsilon m_{e} / m_{\tau}$, where $\epsilon$ measures the $U(1)_{L^{\prime}}$-breaking. Since $\Delta m_{\text {atm }}^{2} \sim 3.5 \times 10^{-3} \mathrm{eV}^{2}$, we find that $\Delta m_{\odot}^{2} \sim \epsilon 10^{-6} \mathrm{eV}^{2}$, which will fall into the allowed region of the LOW solution to the solar neutrino problem for $\epsilon \sim 0.1$.
\end{abstract}

PACS: 12.60.-i, 13.15.+g, 14.60.Pq, 14.60.St

Keywords: neutrino oscillations, neutrino mass, radiative mechanism

Recent evidence for atmospheric neutrino oscillations [1] has promoted new theoretical activities of understanding properties of neutrinos, especially concerning the long-standing theoretical issue of their masses and mixings [2]. It is known that there have been so far two main ideas to account for the smallness of neutrino masses, which are, respectively, called as seesaw mechanism [3] and as radiative mechanism [4,5]. Various possibilities of realizing the radiative mechanism in neutrino physics have been discussed [6 8]. Especially, in recent analyses on neutrino mass matrix of the Zee-type [4], the usefulness of the conserved quantum number of $L_{e}-L_{\mu}-L_{\tau}\left(=L^{\prime}\right)$ [9] has been recognized [8]. This $U(1)_{L^{\prime}}$ symmetry works to yield maximal mixing in both atmospheric and solar neutrino oscillations [10]. The maximal mixing in atmospheric neutrino oscillations has been supported by the data indicating that $\sin ^{2} 2 \theta_{23} \sim 1$ with $\Delta m_{23}^{2} \sim 3.5 \times 10^{-3} \mathrm{eV}^{2}$ 近, where $\theta_{i j}$ stands for the mixing angle and $\Delta m_{i j}^{2}$ stands for the squared mass difference for $\nu^{i} \leftrightarrow \nu^{j}$. Solar neutrino oscillations have also been considered to exhibit the maximal mixing if the oscillations are described by $\Delta m_{12}^{2} \sim 3 \times 10^{-5} \mathrm{eV}^{2}$ as a large mixing angle solution (LMA), $\sim 10^{-7}$ $\mathrm{eV}^{2}$ as a less probable solution with low probability and low mass (LOW) and $\sim 10^{-10} \mathrm{eV}^{2}$ as a vacuum oscillation solution (VO) 11.

In the present article, we further apply the ansätz of the $L^{\prime}$-conservation to models of neutrino masses based on two-loop radiative mechanism [5]. It is anticipated to provide more natural explanation of the tiny neutrino mass without enhanced suppression in couplings, which is experimentally of order $0.01 \mathrm{eV}$ 12]. Furthermore, some flavorchanging interactions receive extra suppression owing to the presence of the approximate $U(1)_{L^{\prime}}$ symmetry. It should be also noticed that, in the radiative mechanism of the Zee type, which is based on one-loop diagrams, fine-tuning of lepton-number violating couplings is necessary to yield bimaximal mixing even if one invokes the $L^{\prime}$-conservation. The fine-tuning can be characterized by "inverse hierarchy in the couplings", namely, $f_{[13]} m_{\tau}^{2} \sim f_{[12]} m_{\mu}^{2}[\mathbb{8}$, where $f$ 's are to be defined in Eq.(1). In the present model, it will be shown that nearly bimaximal structure is dynamically guaranteed by the heaviness of the $\tau$ lepton and by the lightness of the electron. Therefore, no fine-tuning is necessary.

The two-loop radiative mechanism can be embedded in the standard model by employing two $S U(2)_{L}$-singlet charged Higgs scalar, $h^{+}$and $k^{++}$, in addition to the standard Higgs, $\phi$. The extra Higgses, $h^{+}$and $k^{++}$, respectively, couple to charged lepton-neutrino pairs and charged lepton-charged lepton pairs. Their interactions are described by

$$
-\mathcal{L}_{h}=\sum_{i, j=1,2,3} \frac{1}{2} f_{[i j]} \overline{\left(\psi_{L}^{i}\right)^{c}} \psi_{L}^{j} h^{+}+f_{\{i j\}} \overline{\left(\ell_{R}^{i}\right)^{c}} \ell_{R}^{j} k^{++}+\text {(h.c.) }
$$

where $\psi_{L}^{i}$ and $\ell_{R}^{i}(i=1,2,3)$ stand for three families of leptons and the Yukawa couplings, $f$ 's, satisfy $f_{[i j]}=-f_{[j i]}$ and $f_{\{i j\}}=f_{\{j i\}}$. Now, let us introduce the $U(1)_{L^{\prime}}$ symmetry into $\mathcal{L}_{h}$. By envisioning the import of its breaking effect,

\footnotetext{
*E-mail:teruyuki@post.kek.jp

${ }^{\dagger}$ E-mail:yasue@keyaki.cc.u-tokai.ac.jp
} 
we employ an additional $k^{++}$to be denoted by $k^{\prime++}$. The quantum number, $L^{\prime}$, is assigned to be 1 for $\left(\psi_{L}^{1}, \ell_{R}^{1}\right), 0$ for $\left(\phi, h^{+}, k^{++}\right),-1$ for $\left(\psi_{L}^{2,3}, \ell_{R}^{2,3}\right)$ and -2 for $k^{\prime++}$. The ordinary lepton number, to be denoted by $L$, can also be assigned to be 1 for leptons, 0 for $\phi$ and -2 for $\left(h^{+}, k^{++}, k^{\prime++}\right)$.

Yukawa interactions take the form of

$$
\begin{aligned}
-\mathcal{L}_{Y}= & \sum_{i=1,2,3} f_{\phi}^{i} \overline{\psi_{L}^{i}} \phi \ell_{R}^{i}+\sum_{i=2,3}\left(\frac{1}{2} f_{[1 i]} \overline{\left(\psi_{L}^{1}\right)^{c}} \psi_{L}^{i} h^{+}+f_{\{1 i\}} \overline{\left(\ell_{R}^{1}\right)^{c}} \ell_{R}^{i} k^{++}\right) \\
& \left.+f_{\{11\}} \overline{\left(\ell_{R}^{1}\right)^{c}} \ell_{R}^{1} k^{\prime++}+\text { (h.c. }\right),
\end{aligned}
$$

and Higgs interactions are described by self-Hermitian terms composed of $\varphi \varphi^{\dagger}\left(\varphi=\phi, h^{+}, k^{++}, k^{\prime++}\right)$ and by the non-self-Hermitian terms in

$$
V_{0}=\mu_{0} h^{+} h^{+} k^{++^{\dagger}}+\text { (h.c.) }
$$

where $\mu_{0}$ represents a mass scale. This coupling softly breaks the $L$-conservation but preserves the $L^{\prime}$-conservation. To account for solar neutrino oscillations, the breaking of the $L^{\prime}$-conservation should be included and is assumed to be furnished by

$$
V_{b}=\mu_{b} h^{+} h^{+} k^{\prime++\dagger}+\text { (h.c.) }
$$

where $\mu_{b}$ represents a breaking scale of the $L^{\prime}$-conservation. One can instead introduce a neutral Higgs scalar, which spontaneously breaks $U(1)_{L}$ (or $U(1)_{L^{\prime}}$ ) by acquiring vacuum expectation value related to $\mu_{0}$ (or $\left.\mu_{b}\right)$ [13]. However, there necessarily appears a Nambu-Goldstone boson called Majoron, whose coupling to matter should be kept sufficiently small. To avoid having such a dangerous massless Majoron is to include soft $U(1)$-breaking interactions such as Eqs.(3) and (4), which generate its mass of order of the breaking mass scale.

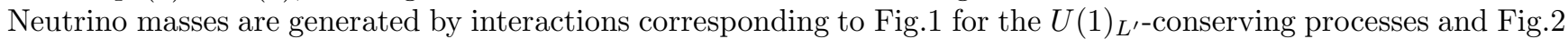

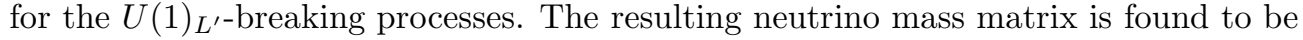

$$
M_{\nu}=\left(\begin{array}{ccc}
0 & m_{12} & m_{13} \\
m_{12} & m_{22}^{\prime} & m_{23}^{\prime} \\
m_{13} & m_{23}^{\prime} & m_{33}^{\prime}
\end{array}\right)
$$

where $m_{1 i}$ and $m_{i j}^{\prime}(i, j=2,3)$ are calculated to be

$$
\begin{aligned}
& m_{1 i}=2 \sum_{j=2,3} f_{[1 j]} f_{\{j 1\}} f_{[1 i]} \frac{m_{\ell_{j}} m_{e} \mu_{0}}{m_{k}^{2}} F\left(m_{\ell_{j}}^{2}, m_{h}^{2}, m_{k}^{2}\right) F\left(m_{e}^{2}, m_{h}^{2}, m_{k}^{2}\right), \\
& m_{i j}^{\prime}=-f_{[1 i]} f_{\{11\}} f_{[1 j]} \frac{m_{e} m_{e} \mu_{b}}{m_{k^{\prime}}^{2}} F\left(m_{e}^{2}, m_{h}^{2}, m_{k^{\prime}}^{2}\right) F\left(m_{e}^{2}, m_{h}^{2}, m_{k^{\prime}}^{2}\right)
\end{aligned}
$$

under the approximation that $m_{k, k^{\prime}}^{2} \gg m_{\ell^{i}, e, h}^{2}$. Mass parameters, $m_{k, k^{\prime}, h}$, respectively, stand for the masses of Higgs scalars, $k^{++}, k^{++}$and $h$, and the function of $F$ is defined by

$$
F(x, y, z)=\frac{1}{16 \pi^{2}} \frac{x \ln (x / z)-y \ln (y / z)}{x-y} .
$$

The outline of its derivation can be seen from the Appendix.

The entries of $m_{12}$ and $m_{13}$ receive contributions from both $\mu$ - and $\tau$-exchange as in Eq.(16). Since $m_{\tau} \gg m_{\mu}$, the $\tau$-exchange gives dominant contributions to $m_{12}$ and $m_{13}$, which result in the same mass-dependence. One can, then, observe that $m_{12} \sim m_{13}$ is a natural consequence without fine-tuning of the couplings. In fact, nearly bimaximal mixing is reproduced by $f_{[12]} \sim f_{[13]}$. Other entries, $m^{\prime}$ 's, are further suppressed by the factor of $m_{e} / m_{\tau}$. Thus, the form of our neutrino mass matrix is consistent with the one described by nearly bimaximal mixing.

We find that $\Delta m_{\text {atm }}^{2}$ for atmospheric neutrino oscillations and $\Delta m_{\odot}^{2}$ for solar neutrino oscillations are calculated to be:

$$
\Delta m_{a t m}^{2}=m_{12}^{2}+m_{13}^{2}\left(\equiv m_{\nu}^{2}\right), \quad \Delta m_{\odot}^{2}=4 m_{\nu} \delta m
$$

with

$$
\delta m=\frac{1}{2}\left|m_{22}^{\prime} \cos ^{2} \theta_{\nu}+2 m_{23}^{\prime} \cos \theta_{\nu} \sin \theta_{\nu}+m_{33}^{\prime} \sin ^{2} \theta_{\nu}\right|,
$$


where the mixing angle $\theta_{\nu}$ is defined by $\cos \theta_{\nu}=m_{12} / m_{\nu}\left(\sin \theta_{\nu}=m_{13} / m_{\nu}\right)$ and the anticipated relation of $\left|m_{i j}^{\prime}\right| \ll$ $\left|m_{1 k}\right|$ for $i, j, k=2,3$ have been used. As far as mass scales are concerned, T1 we reach

$$
\Delta m_{\odot}^{2} \sim \frac{\mu_{b}}{\mu_{0}} \frac{m_{e}}{m_{\tau}} \frac{m_{k}^{2}}{m_{k^{\prime}}^{2}} \Delta m_{a t m}^{2} .
$$

It turns out to be $\Delta m_{\odot}^{2} / \Delta m_{\text {atm }}^{2} \sim \epsilon m_{e} / m_{\tau}$ for $m_{k} \sim m_{k^{\prime}}$, where $\epsilon \sim \mu_{b} / \mu_{0}$, which is the announced result. The experimental value of $\Delta m_{\text {atm }}^{2} \sim 3.5 \times 10^{-3} \mathrm{eV}^{2}$ [1] implies $\Delta m_{\odot}^{2} \sim 10^{-7} \mathrm{eV}^{2}$ for $\epsilon \sim 0.1$, which lies in the region corresponding to the LOW solution to the solar neutrino problem [11].

To see order of magnitude estimates of our parameters, we have to first recognize possible constraints on masses and couplings since the interactions mediated by $h^{+}, k^{++}$and $k^{\prime++}$ disturb the well established low-energy phenomenology. The most stringent constraints on $f$ 's, which are relevant for our discussions, are listed as

$$
\xi\left|\frac{f_{\{11\}} f_{\{12\}}}{\bar{m}_{k}^{2}}\right|<2.9 \times 10^{-11}\left(6 \times 10^{-9}\right) \mathrm{GeV}^{-2},
$$

from $\mu^{-} \rightarrow e^{-} e^{-} e^{+}$with $\mathrm{BR}\left(\mu^{-} \rightarrow e^{-} e^{-} e^{+}\right)<10^{-12}$ 14] $\left(\mu^{-} \rightarrow e^{-}+\gamma\right.$ with $\mathrm{BR}\left(\mu^{-} \rightarrow e^{-}+\gamma\right)<4.9 \times 10^{-11}$ [14]) [15], where $\xi \sim\left(16 \pi^{2}\right)^{-1}\left(\mu_{0} / m_{k}\right)\left(\mu_{b} / m_{k^{\prime}}\right)$ arising from the loop for the $k^{++} k^{\prime++}$ mixing, which represents the extra suppression factor due to $U(1)_{L^{\prime}}$ and $\bar{m}_{k}^{2}$ stands for the averaged mass of $k^{++}$and $k^{\prime++}$, and

$$
\left|\frac{f_{\{11\}}}{m_{k^{\prime}}}\right|^{2}<1.2 \times 10^{-5} \mathrm{GeV}^{-2},
$$

from $e^{-} e^{-} \rightarrow e^{-} e^{-}$16]. The contributions to this process via the $k^{++}$-exchanges turn out to be higher loop-effects since $k^{++}$does not directly couple to $e^{-} e^{-}$and are expected to be well suppressed. The $\mu$ decay of $\mu^{-} \rightarrow \nu_{\mu} e^{-} \bar{\nu}_{e}$ is used to determine the value of the Fermi constant, which includes the extra $h^{+}$-contributions, thus, providing slight deviation of the electroweak gauge coupling of $g$ from the standard value; therefore, the constraint should be deduced from that on $g$ [17, which can be translated into

$$
\left|\frac{f_{[12]}}{m_{h}}\right|^{2}<1.7 \times 10^{-6} \mathrm{GeV}^{-2},
$$

for $\nu_{\mu}\left(\bar{\nu}_{\mu}\right) e^{-} \rightarrow \nu_{\mu}\left(\bar{\nu}_{\mu}\right) e^{-}$.

For the present analysis, the couplings of $f$ 's are kept as small as $\mathcal{O}(e)$. We adopt the following parameter values that satisfy these constraints, where $f_{[12]} \sim f_{[13]}$ is assumed to yield nearly bimaximal mixing: $f_{[12]} \sim f_{[13]} \sim 2 e$ yielding $m_{h}>350 \mathrm{GeV}$ by Eq.(14), from which $m_{h} \sim 350 \mathrm{GeV}$ is taken, $f_{\{11\}} \sim f_{\{13\}} \sim e, m_{k} \sim 2$ TeV with $m_{k}-m_{k^{\prime}} \sim m_{k} / 10$ and $\mu_{0} \sim 1.5 \mathrm{TeV}$ with $\mu_{b} \sim \mu_{0} / 10$ giving $\epsilon \sim 0.1$. The constraint of Eq.(12) is satisfied by $f_{\{12\}}$ $\lesssim 1$. These parameters, in fact, reproduce $\Delta m_{\text {atm }}^{2} \sim 2.4 \times 10^{-3} \mathrm{eV}^{2}$ and $\Delta m_{\odot}^{2} \sim 10^{-7} \mathrm{eV}^{2}$, which is relevant for the LOW solution to the solar neutrino problem. The mass scale of the heaviest neutrino mass is characterized by $\left(16 \pi^{2}\right)^{-2}\left(m_{e} m_{\tau} \mu_{0} / m_{k}^{2}\right) \sim 0.01 \mathrm{eV}$.

To conclude, we have demonstrated that two-loop radiative mechanism well works to account for neutrino oscillation phenomena when it is combined with the $U(1)_{L^{\prime}}$ symmetry. Thanks to the well known loop-factor of $\left(16 \pi^{2}\right)^{-2}$, neutrino masses are well suppressed to yield $\mathcal{O}(0.01) \mathrm{eV}$. The couplings of $f$ 's can be chosen to be $\mathcal{O}(e)$ as $f_{[12]} \sim f_{[13]} \sim 2 e$ and $f_{\{11\}} \sim f_{\{13\}} \sim e$. Solar neutrino oscillations are controlled by the factor of $\left(m_{e} / m_{k^{\prime}}\right)^{2}$, leading to the relation of $\Delta m_{\odot}^{2} \sim\left(m_{e} \mu_{b} / m_{\tau} \mu_{0}\right) \Delta m_{a t m}^{2}$, which provides the LOW solution for $\mu_{b} \sim 0.1 \mu_{0}$.

The work of M.Y. is supported by the Grant-in-Aid for Scientific Research No 12047223 from the Ministry of Education, Science, Sports and Culture, Japan.

Note added: While preparing this manuscript, we are aware of the article [18] that has treated the same subject and has reached slightly different conclusion.

1 The possible contributions to the $\nu_{e}-\nu_{e}$ entry of $M_{\nu}$ in Eq.(周), arising from the three loop diagrams found in Ref. [18], come from four-loop diagrams involving the loop for the $k^{++}-k^{++}$mixing characterized by the factor of $\xi(\sim$ $\left.\left(16 \pi^{2}\right)^{-1}\left(\mu_{0} / m_{k}\right)\left(\mu_{b} / m_{k^{\prime}}\right)\right)$. This diagram at most yields $\delta \sim\left(16 \pi^{2}\right)^{-1} \xi m_{\tau}^{2} / m_{k}^{2}$, which should be compared with $m_{e}^{2} / m_{k^{\prime}}^{2}$. Our estimate of $\Delta m_{\odot}^{2} \propto m_{e}^{2}$ is not drastically altered by including this effect since the present parameter set gives $\delta \sim 2 m_{e}^{2} / m_{k}^{2}$, which turns out to be $\mathcal{O}\left(m_{e}^{2} / m_{k^{\prime}}^{2}\right)$.

${ }^{2}$ Of course, $\epsilon \sim 10^{-4}$ gives $\Delta m_{\odot}^{2} \sim 10^{-10} \mathrm{eV}^{2}$, corresponding to the VO solution [18]. However, it is not suitable for our discussions to obtain a tiny mass-splitting without such enhanced suppression in couplings. 


\section{Appendix}

In this Appendix, we describe the outline of obtaining the integral of Eq.(8) used in Eqs.(6) and (7). From the diagram in Fig.1, we write the relevant integration to be:

$$
I=\int \frac{d^{4} k}{(2 \pi)^{4}} \frac{d^{4} q}{(2 \pi)^{4}} \frac{1}{\left(k^{2}-m_{\ell}^{2}\right)\left(k^{2}-m_{h}^{2}\right)\left(q^{2}-m_{e}^{2}\right)\left(q^{2}-m_{h}^{2}\right)\left((k-q)^{2}-m_{k}^{2}\right)} .
$$

By performing the intgration over $k$ supplemented by

$$
\frac{1}{a b c}=\frac{\Gamma(3)}{\Gamma(1) \Gamma(1) \Gamma(1)} \int_{0}^{1} d x \int_{0}^{1} y d y \frac{1}{[c+(b-c) y+(a-b) x y]^{3}},
$$

and by noticing the formula for the one-loop integral

$$
\int \frac{d^{4} q}{(2 \pi)^{4}} \frac{1}{\left(q^{2}-a\right)\left(q^{2}-b\right)\left(q^{2}-c\right)}=-\frac{i}{16 \pi^{2}}\left[\frac{a \ln a}{(a-b)(a-c)}+\frac{b \ln b}{(b-a)(b-c)}+\frac{c \ln c}{(c-a)(c-b)}\right],
$$

we reach

$$
I=\int d x y d y \frac{i}{16 \pi^{2}[y(1-y)]} I(x, y),
$$

where

$$
I(x, y)=-\frac{i}{16 \pi^{2}}\left[\frac{m_{e}^{2} \ln m_{e}^{2}}{\left(m_{e}^{2}-m_{h}^{2}\right)\left(m_{e}^{2}-M^{2}\right)}+\frac{m_{h}^{2} \ln m_{h}^{2}}{\left(m_{h}^{2}-m_{e}^{2}\right)\left(m_{h}^{2}-M^{2}\right)}+\frac{M^{2} \ln M^{2}}{\left(M^{2}-m_{h}^{2}\right)\left(M^{2}-m_{e}^{2}\right)}\right]
$$

with

$$
M^{2}=\frac{m_{k}^{2}-\left(m_{k}^{2}-m_{h}^{2}\right) y-\left(m_{h}^{2}-m_{\ell}^{2}\right) x y}{y(1-y)} .
$$

Under the approximation of $m_{k}^{2} \gg m_{\ell, e, h}^{2}$, we find that

$$
y(1-y)\left(a-M^{2}\right) \approx-a(y-\alpha)(y-\beta)
$$

with

$$
\alpha=\frac{m_{k}^{2}}{a}\left[1-\frac{m_{h}^{2}-\left(m_{h}^{2}-m_{\ell}^{2}\right) x}{m_{k}^{2}}\right], \quad \beta=1+\frac{m_{h}^{2}-\left(m_{h}^{2}-m_{\ell}^{2}\right) x}{m_{k}^{2}},
$$

which yield

$$
J(a)=\int d x y d y \frac{1}{y(1-y)\left(a-M^{2}\right)} \approx \frac{1}{m_{k}^{2}} \frac{m_{\ell}^{2} \ln \left(m_{\ell}^{2} / m_{k}^{2}\right)-m_{h}^{2} \ln \left(m_{h}^{2} / m_{k}^{2}\right)}{m_{\ell}^{2}-m_{h}^{2}}
$$

and

$$
\int d x y d y \frac{M^{2} \ln M^{2}}{y(1-y)\left(a-M^{2}\right)\left(b-M^{2}\right)} \approx-\frac{a J(a)-b J(b)}{a-b} \ln m_{k}^{2},
$$

where we have used $\ln M^{2} \approx \ln m_{k}^{2}$. The parameters of $a$ and $b$ should satisfy the condition of $a, b \ll m_{k}^{2}$. The function of $J(a)$ turns out to be independent of $a$ in the present approximation. Collecting these results, we finally obtain

$$
I=\frac{F\left(m_{\ell}^{2}, m_{h}^{2}, m_{k}^{2}\right) F\left(m_{e}^{2}, m_{h}^{2}, m_{k}^{2}\right)}{m_{k}^{2}},
$$

where

$$
F(x, y, z)=\frac{1}{16 \pi^{2}} \frac{x \ln (x / z)-y \ln (y / z)}{x-y},
$$

which is the expression of Eq.(8). 
[1] SuperKamiokande Collaboration, Y. Fukuda et al., Phys. Rev. Lett. 81 (1998) 1562; Phys. Lett. B 433 (1998) 9 and 436 (1998) 33. See also K. Scholberg, hep-ex/9905016 (May, 1999). For recent analysis, see N. Forego, M.C. Gonzalez-Garcia and J.W.F. Valle, JHEP 7 (2000) 006; Nucl. Phys. B 580 (2000) 58.

[2] Z. Maki, M. Nakagawa and S. Sakata, Prog. Theor. Phys. 28 (1962) 870. See also B. Pontecorvo, JETP (USSR) 34 (1958) 247; Zh. Eksp. Teor. Piz. 53 (1967) 1717; V. Gribov and B. Pontecorvo, Phys. Lett. 28B (1969) 493.

[3] T. Yanagida, in Proceedings of the Workshop on Unified Theories and Baryon Number in the Universe edited by A. Sawada and A. Sugamoto (KEK Report No.79-18, Tsukuba, 1979), p.95; Prog. Theor. Phys. 64 (1980) 1103; M. Gell-Mann, P. Ramond and R. Slansky, in Supergravity edited by P. van Nieuwenhuizen and D.Z. Freedmann (North-Holland, Amsterdam 1979), p.315; R.N. Mohapatra and G. Senjanovic, Phys. Rev. Lett. 44 (1980) 912.

[4] A. Zee, Phys. Lett. 93B (1980) 389; 161B (1985) 141; L. Wolfenstein, Nucl. Phys. B175 (1980) 93.

[5] A. Zee, Nucl. Phys. B264 (1986) 99; K. S. Babu, Phys. Lett. B 203 (1988) 132; D. Chang, W.-Y.Keung and P.B. Pal, Phys. Rev. Lett. 61 (1988) 2420. See also, J. Schechter and J.W.F. Valle, Phys. Rev. D 25 (1982) 2951.

[6] S. P. Petcov, Phys. Lett. 115B (1982) 401; K. S. Babu and V. S. Mathur, Phys. Lett. B 196 (1987) $218 ;$ J. Liu, Phys. Lett. B 216 (1989) 367; D. Chang and W.-Y. Keung, Phys. Rev. D 39 (1989) 1386; W. Grimus and H. Neufeld, Phys. Lett. B 237 (1990) 521; B.K. Pal, Phys. Rev. D 44 (1991) 2261; W. Grimus and G. Nardulli, Phys. Lett. B 271 (1991) 161; J.T. Polonium, A. Yu. Smirnov and J.W.F. Valle, Phys. Lett. B 286 (1992) 321; A. Yu. Smirnov and Z. Tao, Nucl. Phys. B 426 (1994) 415.

[7] A.Yu. Smirnov and M. Tanimoto, Phys. Rev. D 55 (1997) 1665; N. Gaur, A. Ghosal, E. Ma and P. Roy, Phys. Rev D 58 (1998) 071301; Y. Okamoto and M. Yasuè, Prog. Theor. Phys. 101 (1999) 1119; Phys. Lett. B 466 (1999) 267; G.C. McLaughlin and J.N. Ng, Phys. Lett. B 455 (1999) 224; J.E. Kim and J.S. Lee, hep-ph/9907452 (July, 1999); N. Haba, M. Matsuda and M. Tanimoto, Phys. Lett. B 478 (2000) 351; C.-K. Chula, X.-G. He and W-Y.P. Hwang, Phys. Lett. B 479 (2000) 224; K. Cheung and O.C.W. Kong, Phys. Rev. D 61 (2000) 113012.

[8] C. Jarlskog, M. Matsuda, S. Skadhauge and M. Tanimoto, Phys. Lett. B 449 (1999) 240; P.H. Frampton and S.L. Glashow, Phys. Lett. B 461 (1999) 95; A.S. Joshipura and S.D. Rindani, Phys. Lett. B 464 (1999) 239; D. Chang and A. Zee, Phys. Rev. D 61 (2000) 071303.

[9] R. Barbieri, L.J. Hall, D. Smith, A. Strumia and N. Weiner, JHEP 12 (1998) 017.

[10] D.V. Ahluwalia, Mod. Phys. Lett. A 13 (1998) 2249; V. Barger, P. Pakvasa, T.J. Weiler and K. Whisnant, Phys. Lett. B 437 (1998) 107; A. Baltz, A.S. Goldhaber and M. Goldharber, Phys. Rev. Lett. 81 (1998) 5730; Y. Nomura and T. Yanagida, Phys. Rev. D 59 (1999) 017303; M. Jezabek and Y. Sumino, Phys. Lett. B 440 (1998) 327; R.N. Mohapatra and S. Nussinov, Phys. Lett. B 441 (1998) 299; Q. Shafi and Z. Tavartkiladze, Phys. Lett. B 451 (1999) 129; Phys.Lett. B 482 (2000) 145; I. Stancu and D.V. Ahluwalia, Phys. Lett. B 460 (1999) 431; H. Georgi and S.L. Glashow, Phys. Rev. D 61 (2000) 097301; R. N. Mohapatra, A. Pérez-Lorenzana and C.A. de S. Pires, Phys. Lett. B 474 (2000) 355.

[11] J.N. Bahcall, P. Krastev and A.Yu. Smirnov, Phys. Rev. D 60 (1999) 093001; M.C. Gonzalez-Garcia, P.C. de Holanda, C. Pena-Garay and J.W.F. Valle, Nucl. Phys. B 573 (2000) 3; G.L. Fogli, E. Lisi, D. Montanino and A. Palazzo, Phys. Rev. D 62 (2000) 013002,

[12] See for example, R.N. Mohapatra, hep-ph/9910365 (Oct., 1999).

[13] D. Chang, W.-Y.Keung and P.B. Pal, in Ref. 55.

[14] Particle Data Group, Euro. Phys. J. C 3 (1998).

[15] K. S. Babu, in Ref. [5].

[16] A.S. Joshipura and S.D. Rindani, in Ref. 8]

[17] A.Yu. Smirnov and M. Tanimoto, in Ref. [7].

[18] L. Lavoura, hep-ph/0005321 (May, 2000).

\section{Figure Captions}

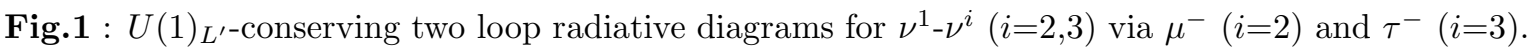

Fig.2 : $U(1)_{L^{\prime}}$-breaking two loop radiative diagrams for $\nu^{i} \nu^{j}(i, j=2,3)$ via $e^{-}$. 


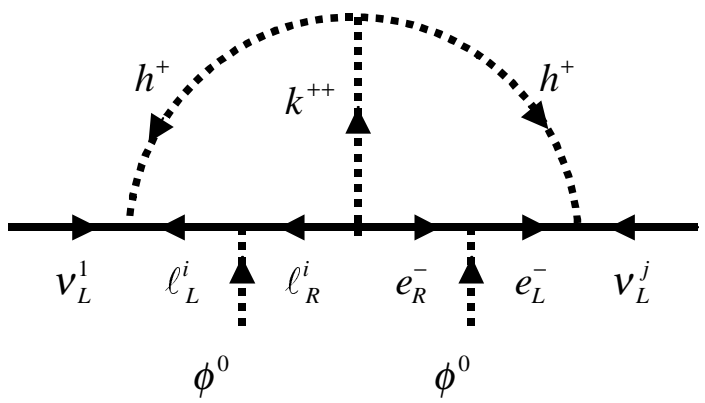

Fig. 1: $U(1)_{L^{\prime}}$-conserving two loop radiative diagrams for $v^{1}-v^{j} \quad(j=2,3)$ via $\mu^{-} \quad(i=2)$ and $\tau^{-}(i=3)$.

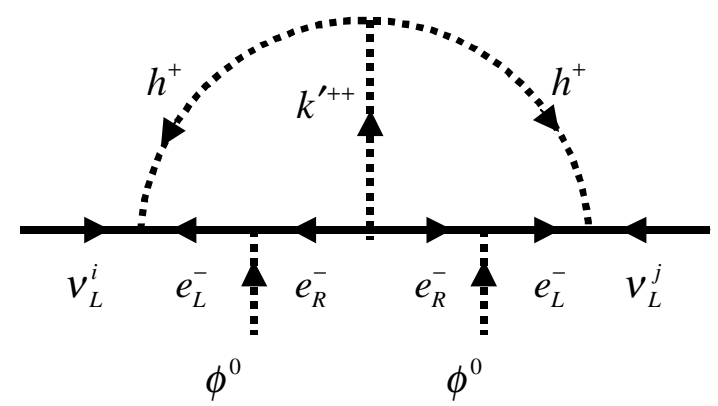

Fig. 2: $U(1)_{L^{\prime}}$-breaking two loop radiative diagrams for $v^{i}-v^{j} \quad(i . j=2,3)$ via $e^{-}$. 